\title{
Case report of modified Box and Blocks test with motion capture to measure prosthetic function
}

\author{
Jacqueline S. Hebert, MD, FRCPC; ${ }^{*}$ Justin Lewicke, MBA, BSc(Kin) $^{2}$ \\ ${ }^{1}$ Division of Physical Medicine \& Rehabilitation, University of Alberta, Edmonton, Alberta, Canada, and Adult Amputee \\ Program, Glenrose Rehabilitation Hospital, Edmonton, Alberta, Canada; ${ }^{2}$ Syncrude Centre for Motion and Balance, Glen- \\ rose Rehabilitation Hospital, Edmonton, Alberta, Canada
}

\begin{abstract}
This case study report demonstrates the use of motion analysis with a modification of the Box and Blocks test. The goal was to quantify observed improvements in compensatory movements and simultaneous control in a subject using different prostheses before and after targeted muscle reinnervation (TMR) surgery. This is a single case study with data collection using a body-powered prosthesis pre-TMR surgery and 6 mo postfitting with a TMR myoelectric prosthesis. The Box and Blocks test was modified for cyclical motion within a motion capture laboratory. With the TMR myoelectric prosthesis, the subject was able to simultaneously activate the hand and elbow. Task performance was slower, but there was improved elbow flexion and less trunk compensatory motion than with the body-powered prosthesis. There are several limitations to the case study because there is no direct comparison of myoelectric performance before and after TMR surgery; however, the current report presents a potential method to quantify quality of motion and compensatory movements of prosthetic users. With further study, this test procedure has the potential to be a useful outcome measure for future standardized assessments of upper-limb prosthetic function.
\end{abstract}

Key words: biomechanics, kinematics, motion, movement, outcome and process assessment, outcome measures, rehabilitation outcome, traumatic amputation, treatment outcome, upper limb.

\section{INTRODUCTION}

Advances in prosthetic design and treatment interventions to improve outcome after upper-limb amputation are expanding faster than the ability to measure their effectiveness. There is a noticeable movement to improve and standardize upper-limb prosthetic outcome measures [1]. This movement includes emphasis on the use of the World Health Organization's International Classification of Functioning, Disability, and Health model [2] to quantify and fully understand the effect of upper-limb loss and to assist in determining what treatments effectively minimize activity limitations and participation restrictions.

A variety of outcome tools are recommended to measure activity and participation outcomes following upperlimb amputation [3]. However, basic functional performance measures are still the basis of much clinical decision-making because the results are immediately evident for the clinician. Given this, we are faced with an array of functional outcome measures that have been designed for upper-limb impairments but not always specifically for prosthetic users [4]. One such measure of gross manual upper-limb function is the Box and Blocks test. The Box and Blocks test is a specific performance task testing gross manual dexterity of the upper limb [5].

\footnotetext{
Abbreviations: $\mathrm{C} 7=$ seventh cervical spinous process, $\mathrm{TH}=$ transhumeral, TMR = targeted muscle reinnervation.

*Address all correspondence to Jacqueline S. Hebert, MD, FRCPC; University of Alberta, Division of Physical Medicine \& Rehabilitation, Glenrose Rehabilitation Hospital, 10230-111 Avenue, Edmonton, Alberta, Canada T5G 0B7; 780-735-8870; fax: 780-735-6018.
}

Email: jhebert@ualberta.ca

http://dx.doi.org/10.1682/JRRD.2011.10.0207 
The test measures quantity of blocks moved in 1 minute, regardless of quality of movement, and is a widely used outcome measure of upper-limb function [6-8] that is commonly available in rehabilitation centers. It is easily set up, requires minimal equipment, and can be performed in a seated position, making it appropriate for multiple diagnostic groups. The Box and Blocks test has been used to examine upper-limb prosthetic function following amputation and to examine myoelectric control after targeted muscle reinnervation (TMR) surgery [9-11]. Although the Box and Blocks test has not been validated specifically with upperlimb prosthetic users, it would seem to have potential for use in this population.

However, the goal of restoring optimal upper-limb prosthetic function typically includes not only improving quantitative performance but also improving quality of motion, specifically smoothness of the pattern of motion of the prosthesis and the ability to target and control excursion of prosthetic grasp [12-13]. In addition to studying motion of the prosthesis, it is important to consider the compensatory motions of the user's body that are required to adapt to the limited degrees of freedom of a prosthetic device. With advances in upper-limb management, such as TMR surgery, accurately quantifying the effect of interventions is essential because observations on the quality of movement can be more impressive than timed tasks or traditional outcome measures of upper-limb function. For example, Miller et al. reported "powered humeral rotation allowed dynamic control of a full range of motion. It allowed the subject to reduce compensatory movements, such as moving his torso, and to stand in a fixed position" [14, p. 2061]. Other reported advantages of TMR myoelectric use following reinnervation surgery include the ability to simultaneously control multiple degrees of freedom of powered components [9,14-15], in contrast to body-powered and standard myoelectric prostheses, which require sequential activation of each joint and thereby control one degree of freedom at a time. Improved simultaneous control is expected to reduce compensatory trunk and shoulder movements required for functional upper-limb tasks and improve performance, but this has not been definitively demonstrated.

One method of examining quality of motion and compensatory motion is quantitative motion analysis. Upperlimb motion analysis is not novel to the prosthetic population [16], but there is a lack of consensus in defining a standardized task or methodology to allow comparison and consistency across investigators. More recently, stan- dardized methods for upper-limb functional tasks have been proposed by various authors [17-19] in order to define an upper-limb motion analysis protocol analogous to lowerlimb gait analysis. There has also been a move to standardize use of the marker set and joint coordinate system set forth by the International Society of Biomechanics standard [20], although this has not been universally adopted. One proposed method of using upper-limb kinematic analysis is to partner it with commonly used functional task performances in a standardized fashion. Although functional tasks have been suggested for motion capture [17], the complexity of the task and requirement for consistency of performance remains a limiting factor. With a specific repetitive task such as the Box and Blocks test, the motion of the prosthetic limb can be repeatedly observed and recorded. The cyclical nature of the task makes it ideal for motion capture [21]. For this reason, in our center we have tried a method not previously reported of using a modified Box and Blocks test with motion capture in order to compare the performance of a subject using different prosthetic devices.

The purpose of this report is to present our case study demonstrating the use of motion analysis, in combination with a modified Box and Blocks test, to quantify the observed improvements in compensatory movements and simultaneous control in a subject using two different prostheses: a body-powered prosthesis and a TMR myoelectric prosthesis. We hope that this case study illustrates a potential method for future standardized assessments of upper-limb prosthetic function that takes into account not only the motion of the prosthetic device but also the function and movement of the individual user.

\section{METHODS}

This report is a retrospective single case study review.

\section{Subject}

A 28-year-old male underwent traumatic left transhumeral (TH) amputation and left knee disarticulation amputation on July 4, 2006. The subject's left TH amputation healed uneventfully, and 6 wk postamputation he was fit with a body-powered prosthesis with mechanical voluntary opening hook and standard internal locking mechanical elbow with dual control cable (Table 1). The subject underwent TMR surgery 20 mo postamputation, involving reinnervation of median nerve to medial biceps and distal radial nerve to lateral triceps, as per the technique of Kuiken et al. 
Table 1.

Prosthetic components supplied.

\begin{tabular}{|c|c|}
\hline Body-Powered Prosthetic Components & $\begin{array}{c}\text { Targeted Muscle Reinnervation Myoelectric Prosthetic } \\
\text { Components }\end{array}$ \\
\hline - Ohio Willow Wood (Mount Sterling, Ohio) Alpha Locking Liner & - Ohio Willow Wood Alpha Liner (with embedded electrodes) \\
\hline - Otto Bock (Duderstadt, Germany) Ergo Arm elbow & $\begin{array}{l}\text { - Liberating Technologies, Inc. (Holliston, Massachusetts) Boston } \\
\text { elbow }\end{array}$ \\
\hline $\begin{array}{l}\text { - Hosmer (Campbell, California) 5XA aluminum hook and quick } \\
\text { change wrist }\end{array}$ & $\begin{array}{l}\text { - Motion Control, Inc. (Salt Lake City, Utah) Electric Terminal } \\
\text { Device and quick disconnect wrist }\end{array}$ \\
\hline
\end{tabular}

[9]. Following the surgery, the subject resumed wearing his body-powered prosthesis. Reinnervation twitches in the muscles were first noted 4 mo postoperatively, and the subject underwent myoelectric site testing at that time. Instructions were given for ongoing muscle strengthening exercises to improve muscle signals and encourage discrete control of muscle activity. Prosthetic myoelectric fitting began 7 mo postsurgery, and the final TMR myoelectric prosthesis was dispensed within 2 mo (Table 1), with foursite muscle control for elbow flexion (lateral biceps), elbow extension (medial triceps), hand close (medial biceps), and hand open (lateral triceps). Within the first week of fitting, the subject was able to naturally activate the motions of the myoelectric elbow and hand. However, several months of training and acclimatization to the device were required. Specifically, the subject experienced muscle pain and tenderness after a full day of use, thought to be due to muscle fatigue, which would resolve with a day of nonuse of the myoelectric prosthesis. There was also some inconsistency of activation, with involuntary movements noted related to cocontraction of muscles within the socket, likely related to the changing sensitivity of the electrode settings, which would respond to threshold adjustments by his prosthetist. For these reasons, the typical wearing pattern of the subject at the 6 mo post-TMR myoelectric fitting follow-up was to use his TMR myoelectric prosthesis daily for work ( $5 \mathrm{~d}$ per wk), no prosthesis at home in the evening, and a bodypowered prosthesis for specific tasks involving environments with high vibration or dust.

The Box and Blocks test was modified to facilitate the quantitative collection of motion analysis data. The outcome data were recorded at two time points: (1) with the body-powered prosthesis prior to TMR surgery and (2) 6 mo after fitting with the TMR myoelectric device powered by the reinnervated muscles (13 mo post-TMR surgery).

\section{Motion Analysis}

\section{Coordination Tasks Marker Set}

In order to demonstrate simultaneous motion of the prosthetic elbow and terminal device, four markers were used at the following locations: lateral prosthetic elbow hinge, wrist, nonmobile side of the prosthetic hook, and mobile side of the prosthetic hook. Since only one side of the hook moves, the marker added to the mobile side allowed the hook-wrist-elbow angle to be calculated for the purpose of assessing specific movement coordination tasks. Motion analysis trials were initially performed for the motions of simultaneous elbow flexion and hand open, elbow extension and hand close, elbow flexion and hand close, and elbow extension and hand open as per Miller et al. [14] in order to demonstrate simultaneous control of prosthetic joints using myoelectric signals of the reinnervated muscles.

\section{Box and Blocks Marker Set}

To generate the kinematic graphs for the Box and Blocks test, a total of six markers were applied to the subject: sternum, seventh cervical spinous process (C7), acromion (bilaterally), lateral elbow hinge, and wrist. Two markers were placed on the box and one on the top of the divider (Figure 1) in order to identify the location of the box in the virtual laboratory space, as well as the subject's location relative to the box. The triangle of C7, sternum, and acromion gave the orientation and position of the shoulder girdle segment. The single marker on the elbow allowed location of the lateral elbow relative to the orientation and position of the shoulder girdle. The single wrist marker allowed the location of the wrist and enabled calculation of the "pseudo-elbow flexion" angle of the prosthesis. This pseudo-elbow flexion angle of the prosthesis was defined as the angle between three points: wrist, lateral elbow hinge, and acromion. Trunk anterior/posterior tilt 


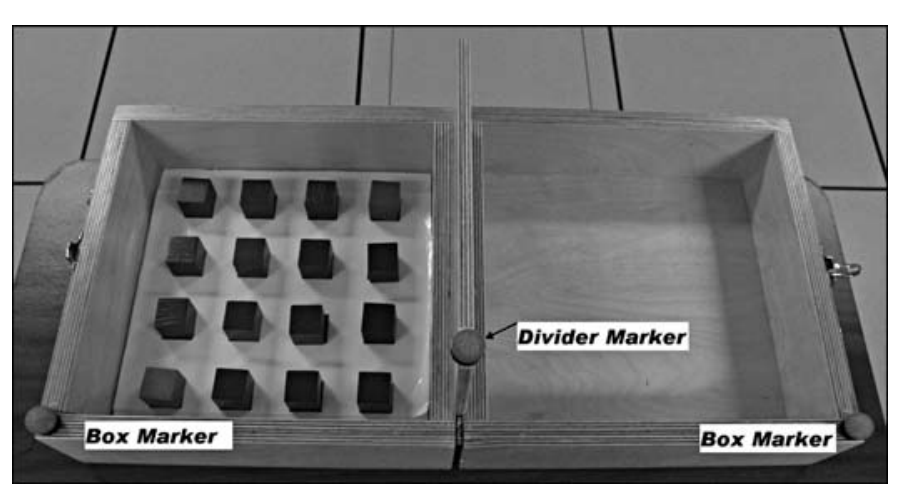

Figure 1.

Standardized placement of blocks for modified Box and Blocks test.

was defined by tilt of the sternum and C7 markers in a vertical plane. The wrist marker was used as the distal tracking point of the terminal device ("terminal marker") because it is the most distal point of the forearm segment that does not move with opening and closing of the terminal device. Two measures were reported from this terminal marker: the altitude relative to the top of the divider (along the $Z$-axis), and the lateral distance from the midline of the subject. Midline was defined as a vertical plane bisecting the sternum and C7 markers. For calculation of the altitude of the terminal marker, because the divider marker was located at the top of the divider (Figure 1) this was referenced as zero, so that negative results indicated the terminal marker below the level of the divider (as the block was picked up), and positive results were the distance above the divider when moving the block over the divider to the other side of the box. The modified Box and Blocks test was also performed with a nonamputee control subject for comparison.

Motion was captured using eight Motion Analysis Corporation (Santa Rosa, California) cameras. These cameras use visible red light and sample at a frequency of $60 \mathrm{~Hz}$. Specific kinematic parameters analyzed comprised trunk medial/lateral shift relative to the divider, trunk pitch relative to the horizon ("trunk anterior/posterior tilt"), prosthetic pseudo-elbow flexion, prosthetic terminal marker location relative to midline of the trunk, and terminal marker location relative to top of the divider.

\section{Modification to Box and Blocks Test}

The Box and Blocks test was chosen for motion analysis because it requires repetitive cycles of motion, allowing for multiple recording trials. However, the random nature of block selection in the standard Box and Blocks test and the placement of the blocks both needed to be addressed in order for us to record and compare multiple trials. Therefore, the placement and order of blocks in the tray to be moved were standardized to 16 blocks placed in 4 rows (Figure 1). The subject was instructed to proceed from the lower inner corner block across the row and then to proceed to the next row. This set-up required specific targeting of the terminal device and consistent activation and arc of movement amenable to motion analysis. In addition to the kinematics of the prosthesis and trunk motion, we recorded the time to complete moving all 16 blocks or the number of blocks moved within $1 \mathrm{~min}$, whichever the subject accomplished first.

The subject stood for the trials, and the top of the table was at the level of the subject's anterior superior iliac spine. This allowed a comfortable range of operation of the prosthetic elbow, with less than complete elbow extension required to pick the block up. The subject was allowed one practice trial, and then three trials were recorded with motion analysis.

\section{RESULTS}

Timed tasks for the Box and Blocks tests are shown in Table 2. The speed of block movement was much slower with the myoelectric than the body-powered prosthesis (less than half). This may be a factor of the subject being an experienced body-powered prosthetic user as his first device versus the more recently fitted TMR myoelectric prosthesis. There was slight variability in the test results; of the three trials of the modified test, the time to move the 16 blocks for each trial was 38, 25, and $25 \mathrm{~s}$ with the body-powered prosthesis and 60,52 , and $56 \mathrm{~s}$ with the myoelectric prosthesis. With the modified test,

Table 2.

Results of standard and modified tests for Box and Blocks.

\begin{tabular}{lcc}
\hline \multicolumn{1}{c}{ Prosthetic } & $\begin{array}{c}\text { Box and Blocks } \\
\text { (No. of blocks } \\
\text { moved) }\end{array}$ & $\begin{array}{c}\text { Modified Box and } \\
\text { Blocks (time to move } \\
\text { 16 blocks [s]) }\end{array}$ \\
\hline Body-Powered & 49 & 29 \\
TMR Myoelectric & 20 & 56 \\
\hline${ }^{*}$ Average of 2 trials; blocks moved in 60 s as per standard Box and Blocks test. \\
${ }^{\dagger}$ Average of 3 trials; time to move 16 blocks of modified Box and Blocks test \\
with motion capture. \\
TMR = targeted muscle reinervation. \\
\hline \hline
\end{tabular}


the lower inner corner block was the most difficult for the subject to access, which resulted in greater delay for the first trial and a possible practice effect for the following two trials. With the standard Box and Blocks test, 53 and 46 blocks were moved using the body-powered prosthesis and 21 and 19 blocks with the myoelectric prosthesis for each of two trials.

The results of the motion analysis when the subject was asked to demonstrate activation of four sequences to track simultaneous control of reinnervated sites using the TMR myoelectric prosthesis are shown in Figure 2. These results demonstrate that the subject was able to activate elbow extension and flexion with hand close simultaneously; however, there was more delay with hand opening maneuvers in combination with elbow motion. This was consistent with the observed clinical prosthetic performance because of weaker hand open muscle signal from lateral triceps.

For the modified Box and Blocks test with motion analysis, we looked at both prosthetic movement (pseudoelbow flexion, terminal marker altitude relative to divider) as well as compensatory motions of the trunk (trunk anterior/posterior tilt). Results are shown in Figures $\mathbf{3}$ to $\mathbf{5}$ and Table 3. The presented figures show one representative entire modified Box and Blocks trial, from start to release of the final block. After release of the final block, there was no standardized position of return, causing inconsistency in the data after release of the last block, which motivated us to truncate the trials to the estimated time of release of the final block. Future studies would benefit from requiring the subject to return to a standard position after completing the test.

The range of elbow flexion with the myoelectric prosthesis was close to that of a nonamputee control subject, in comparison to the static locked elbow used with the bodypowered prosthesis (Table 3). Terminal marker altitude relative to the top of the divider was greatest for the myoelectric prosthetic trial, and a "double bump" pattern was observed only with the myoelectric prosthesis (Figure 4). We believe that an "overshooting" of elbow flexion is responsible for this double bump pattern as the subject makes a correction while shifting the block over to the other side of the box. The amount of trunk anterior/posterior shift was negligible for the control (nonamputee) subject, increased with the body-powered prosthesis to about $26^{\circ}$ total range, and about half that with the myoelectric prosthesis. Other parameters examined (medial-lateral trunk shift and terminal marker midline crossing) did not show noticeable differences between the body-powered and myoelectric prosthesis trials.

From a qualitative perspective, although no formal questionnaire was used, the subject reported feeling increased naturalness of movement and less mental effort to operate the TMR myoelectric prosthesis compared to the body-powered prosthesis.
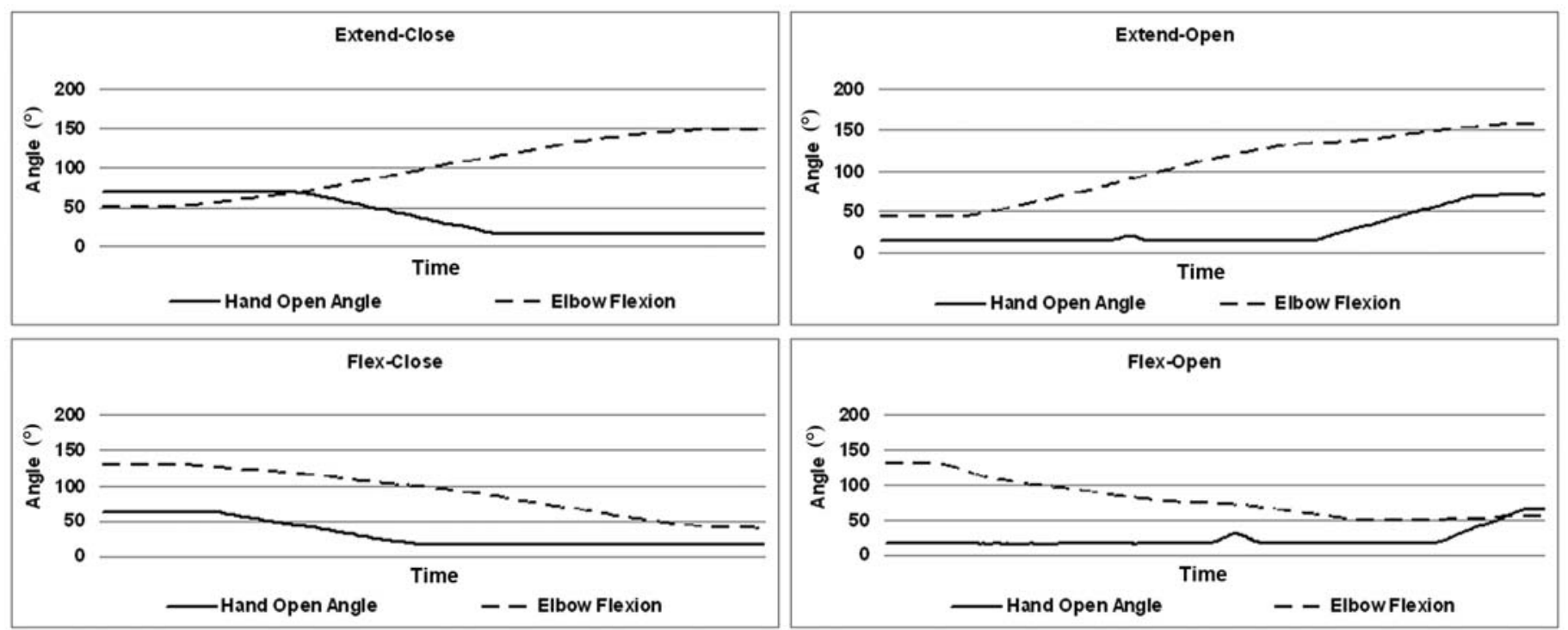

Figure 2.

Kinematics of prosthetic-limb motion showing simultaneous activation of elbow and terminal device. 

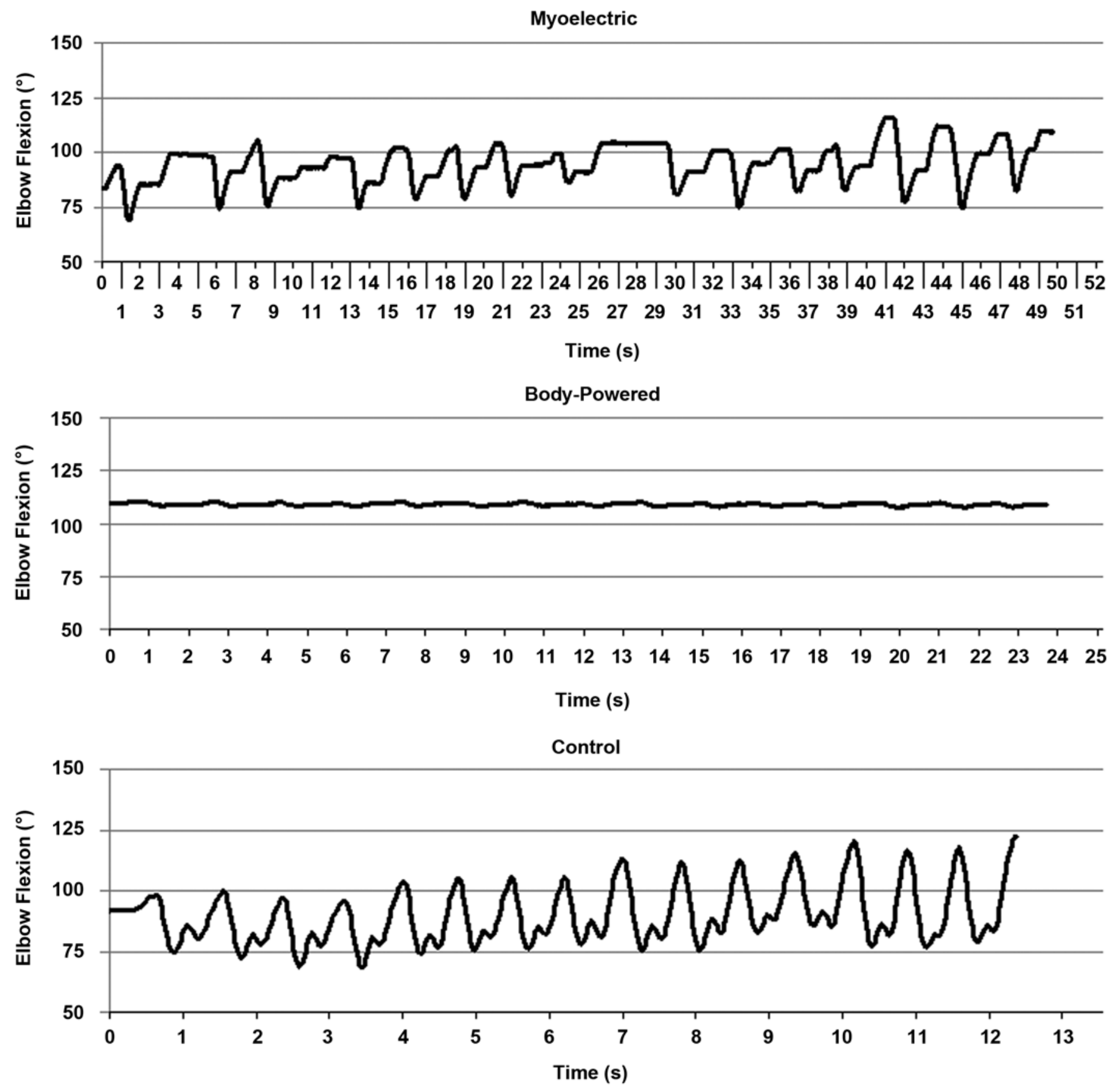

Figure 3.

Elbow flexion angle with modified Box and Blocks test.

\section{DISCUSSION}

The Box and Blocks test is a commonly applied outcome measure for upper-limb function that has established reliability and validity data and standard adult norms [5], although it has not been specifically validated in a population of upper-limb prosthetic users. The current study presents a novel approach to quantifying the quality of prosthetic motion and the compensatory body adjustments required by a transhumeral prosthetic user performing a modified Box and Blocks test, with a comparison of two different prostheses before and after TMR surgery. 

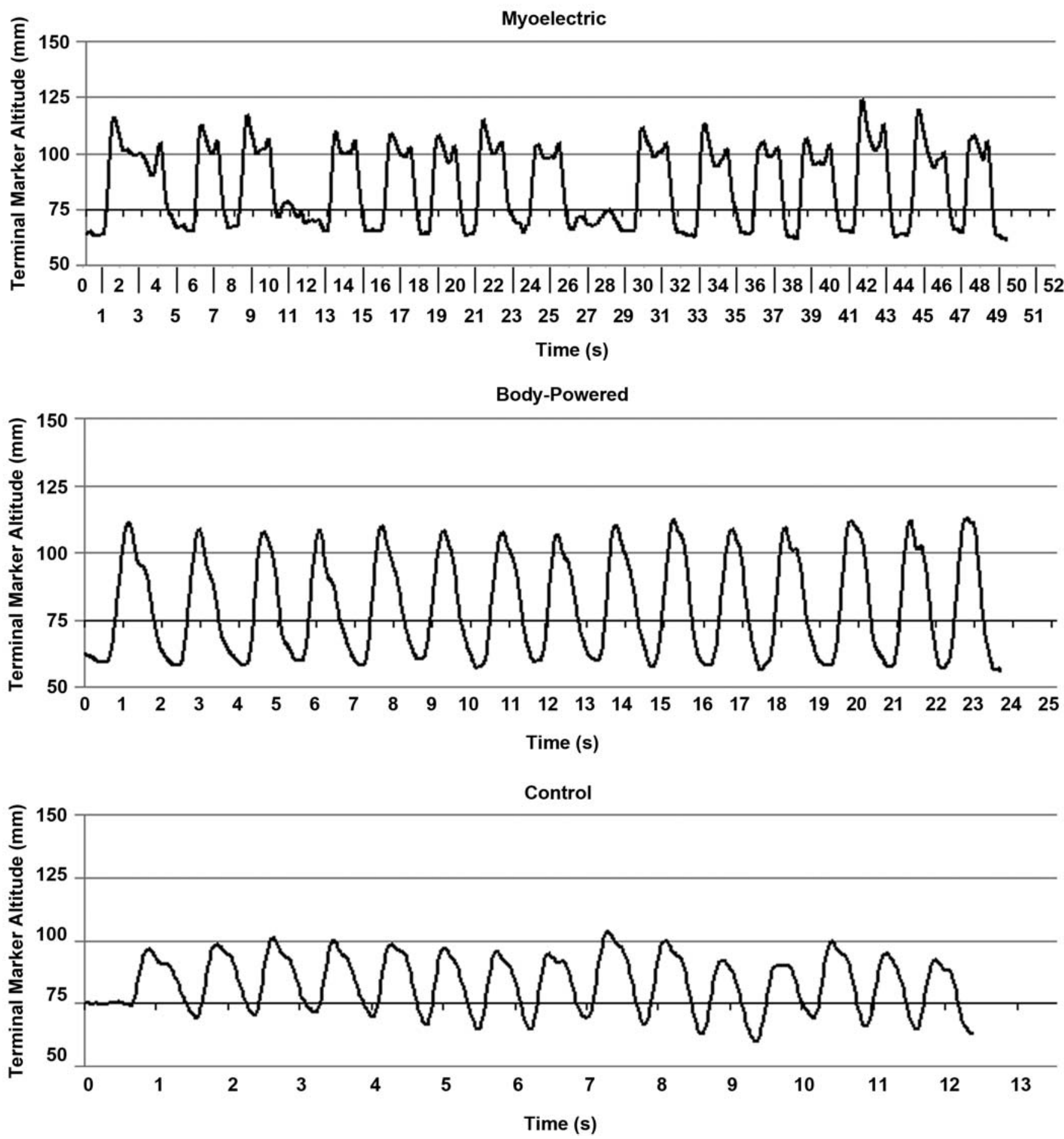

Figure 4.

Terminal marker altitude in relation to divider with modified Box and Blocks test.

Although the Box and Blocks test is simple, easy to construct and administer, and appropriate for a wide range of upper-limb impairments, there are limitations for prosthetic users. Performance of the test in the seated position can limit the range of elbow excursion and has been noted to be difficult for prosthetic users depending 

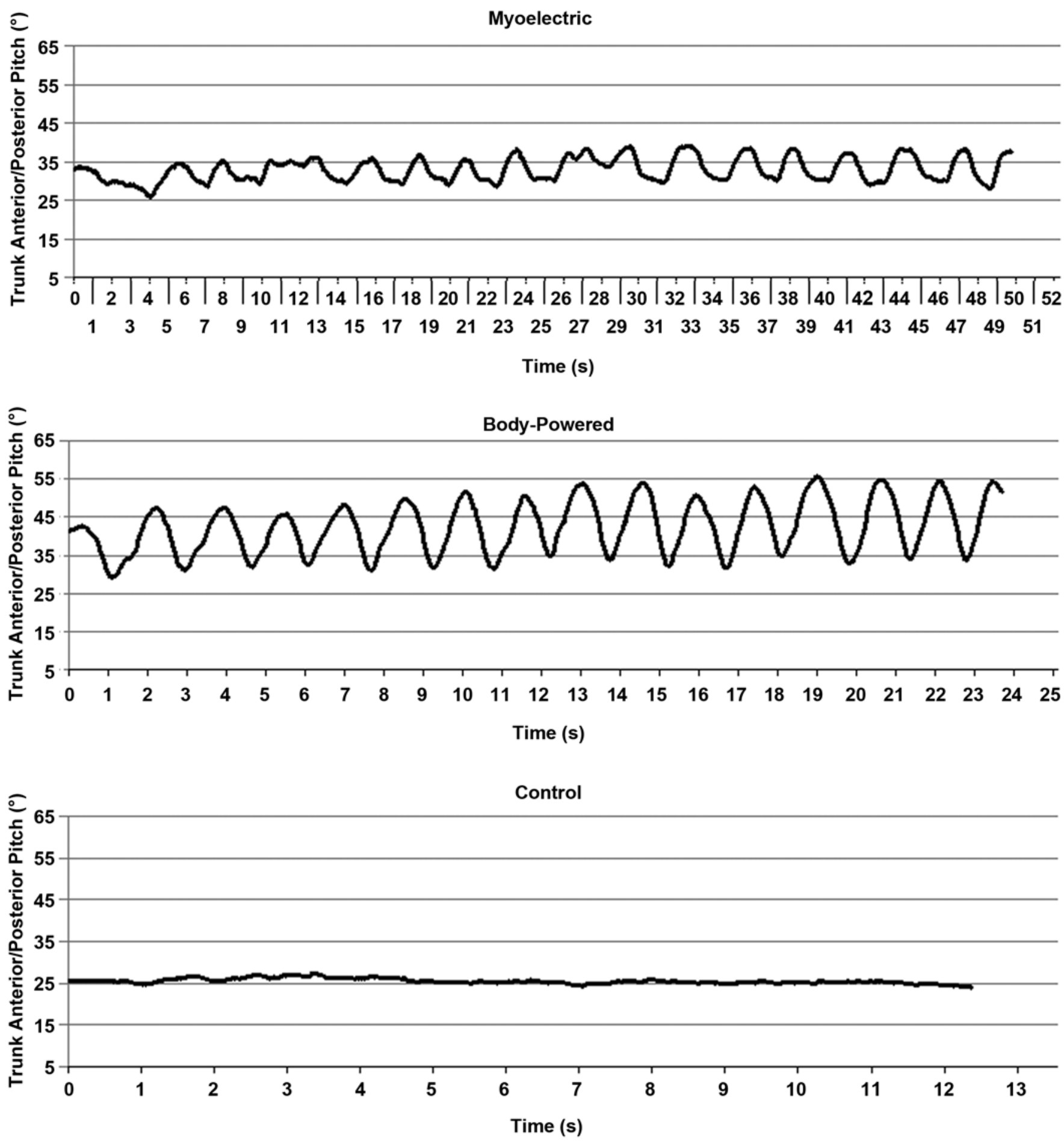

Figure 5.

Trunk anterior/posterior pitch with modified Box and Blocks test.

on the height of the table, which is typically described as "standard height" [5]. The modified Box and Blocks test was chosen in the standing position for our prosthetic user because of this limitation. The consistency of using the same pre- and posttest procedure allowed us to compare results for this single subject; however, further study 
Table 3.

Kinematics of modified Box and Blocks test performance.

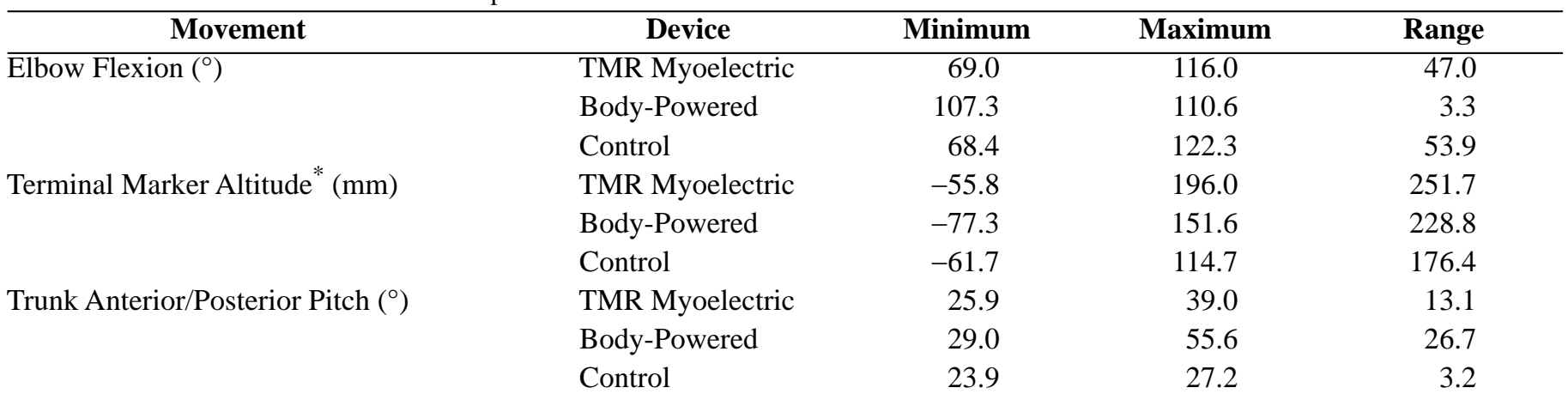

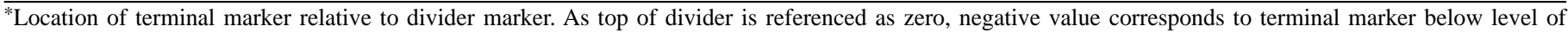
divider marker.

TMR = targeted muscle reinnervation.

will be needed to determine if there are differences in performance expected for a standing versus seated testing procedure. Other factors, such as height of the table, sequence of block movement, and start and end position, will also need to be controlled if normative data ranges are to be established to allow comparison between prosthetic devices and interventions.

In the case study presented, one of the more striking findings is that, while the subject clearly had slower performance with the myoelectric prosthesis, the movement was better and less trunk compensatory adjustments were required to perform the test. From a functional perspective, the slowed speed may be an indication of the subject being only 6 mo postfitting with his first myoelectric prosthesis, compared to the body-powered prosthesis that he was initially trained with and was using daily for over a year prior to his myoelectric fitting. However, faster functional performance when using a prosthesis with the elbow locked may explain why some patients choose mechanical prostheses for simple gross motor tasks instead of myoelectric devices.

The findings in this case study may be related solely to the fact that the elbow was locked in the first trial and that using the myoelectric prosthesis with the elbow locked would have shown similar compensatory movements as with the locked elbow body-powered prosthesis trial. However, the subject preferred to do the task with his elbow locked when using the body-powered prosthesis because he felt this was how he would normally perform the task. We do not think it insignificant to point out that we allowed the patient to perform the task as he would naturally choose rather than artificially imposing a restriction that he would not normally choose as his optimal function. It has been previously suggested that prosthetic users are more consistent in the way they perform tasks than nonprosthetic users [16]. This would suggest that in outcome studies, there is some value to allowing subjects to use the method that they would typically choose in an unobserved environment to present what they see as their best possible performance. Recognizing that testing in a laboratory is an artificial situation, this may allow us to more naturally observe abnormal body biomechanics that the individual might choose to use in the real world. This approach is consistent with current outcome measures such as the Assessment of Capacity for Myoelectric Control [22], which rates a patient's use of the myoelectric prosthesis while performing typical daily tasks.

The subject in this case reported feeling increased naturalness of movement and less mental effort to operate the TMR myoelectric prosthesis compared to the body powered prosthesis. Other authors have demonstrated that upper-limb kinematics of traditional prosthetic function are less smooth with frequent decoupling of the reach and grasp functions and have postulated that focusing on movement pattern may be the key to improving prosthetic use [12]. Excessive body motions in other planes, such as trunk bending [23] and shoulder flexion [24], are also typically performed in order to accomplish prosthetic tasks. The reduced compensatory motions observed in the current study are consistent with studies suggesting that, despite slower function, myoelectric prostheses are preferred to body-powered because of improved range of movement and less compensation required [25]. 
Because this is a case study, we were unable to present comparison of a wide range of prosthetic devices since we were limited to the devices that the subject was provided on a clinical basis. Ideally, this case study would have collected pre-TMR data using a conventional myoelectric prosthesis, but because of funding restrictions the myoelectric prosthesis was not supplied until after the TMR surgery. In the future, direct comparison of myoelectric prosthetic function pre- and post-TMR surgery will be insightful for understanding whether the reduced compensatory movements are related to use of the myoelectric elbow alone or are a benefit of the TMR surgery. The method presented of using motion capture with a modified Box and Blocks test could also allow comparison of different devices in future controlled studies.

Further study would be required to establish normative data for this modified Box and Blocks test with motion capture, including a standardized protocol. Collecting normative data may allow a range of normal function to be identified as a marker for "good quality" motion. Using motion capture in this way has the advantage of allowing examination of the interaction of the users with the device, how they choose to perform with the prosthesis, and how their body adapts to that choice. This may point to a future study on the effect of body compensatory movements on long-term outcomes, such as risk of joint degeneration, muscle overload, and repetitive strain injury, in order to guide selection of the most important parameters to "normalize" in our patients.

In this manner, the motion capture procedure could have wider applicability in other populations with upperlimb impairment for which the Box and Blocks test is commonly used. The traditional Box and Blocks test provides important information on functional upper-limb ability. The addition of quantitative motion analysis has the potential to add to the understanding of quality of movement in upper-limb impairment and to be a relevant outcome measure for interventions to improve upperlimb prosthetic function.

\section{CONCLUSIONS}

The current study presents a novel approach to quantifying quality of motion with a modification of the Box and Blocks test and the use of motion capture in a transhumeral prosthetic user at two time points and with two different prostheses, pre- and post-TMR surgery. We saw improvements in prosthetic motion and reduced compensatory trunk motions, but with slower test performance in the post-TMR myoelectric condition. With further study, the modified Box and Blocks test with motion capture has the potential be a useful standardized outcome measure for a variety of upper-limb impairments because of its ability to quantify motion patterns of the upper limb as well as compensatory body movements.

\section{ACKNOWLEDGMENTS}

\section{Author Contributions:}

Study concept and design: J. S. Hebert, J. Lewicke. Acquisition of data: J. S. Hebert, J. Lewicke.

Analysis and interpretation of data: J. S. Hebert, J. Lewicke.

Drafting of manuscript: J. S. Hebert.

Critical revision of manuscript for important intellectual content:

J. Lewicke.

Financial Disclosures: The authors have declared that no competing interests exist.

Funding/Support: This material was unfunded at the time of manuscript preparation.

Additional Contributions: We would like to thank Vance Pilipchuk for data processing and analysis, Beth Watkins of the Syncrude Centre for Motion and Balance for assistance with data collection, and the Glenrose Rehabilitation Hospital for in-kind support.

Institutional Review: Consent was obtained from the subject for publication of data as per requirements of the Health Ethics Research Board of the University of Alberta.

Participant Follow-Up: The authors plan to inform the participant of the publication of this article.

\section{REFERENCES}

1. Hill W, Kyberd P, Hermansson LN, Hubbard S, Stavdahl $\varnothing$, Swanson S. Upper limb prosthetic outcome measures (ULPOM): a working group and their findings. J Prosthet Orthot. 2009;21(9):69-82. http://dx.doi.org/10.1097/JPO.0b013e3181ae970b

2. World Health Organization. Towards a common language for functioning, disability and health: ICF Beginner's Guide (WHO/EIP/GPE/CAS/01.3). Geneva (Switzerland): WHO; 2002.

3. Miller LA, Swanson S. Summary and recommendations of the Academy's State of the Science Conference on upper limb prosthetic outcome measures. J Prosthet Orthot. 2009;21(9): 83-89. http://dx.doi.org/10.1097/JPO.0b013e3181ae974d

4. Wright V. Prosthetic outcome measures for use with upper limb amputees: a systematic review of the peer-reviewed literature, 1970-2009. J Prosthet Orthot. 2009;21:3-63. http://dx.doi.org/10.1097/JPO.0b013e3181ae9637 
5. Mathiowetz V, Volland G, Kashman N, Weber K. Adult norms for the Box and Block Test of manual dexterity. Am J Occup Ther. 1985;39(6):386-91. [PMID:3160243] http://dx.doi.org/10.5014/ajot.39.6.386

6. Desrosiers J, Bravo G, Hébert R, Dutil E, Mercier L. Validation of the Box and Block Test as a measure of dexterity of elderly people: reliability, validity, and norms studies. Arch Phys Med Rehabil. 1994;75(7):751-55. [PMID:8024419]

7. Mendes MF, Tilbery CP, Balsimelli S, Moreira MA, Cruz AM. [Box and block test of manual dexterity in normal subjects and in patients with multiple sclerosis]. Arq Neuropsiquiatr. 2001;59(4):889-94. Portuguese. [PMID:11733833] http://dx.doi.org/10.1590/S0004-282X2001000600010

8. Canny ML, Thompson JM, Wheeler MJ. Reliability of the box and block test of manual dexterity for use with patients with fibromyalgia. Am J Occup Ther. 2009;63(4):506-10. [PMID:19708480] http://dx.doi.org/10.5014/ajot.63.4.506

9. Kuiken TA, Miller LA, Lipschutz RD, Lock BA, Stubblefield K, Marasco PD, Zhou P, Dumanian GA. Targeted reinnervation for enhanced prosthetic arm function in a woman with a proximal amputation: a case study. Lancet. 2007;369(9559):371-80. [PMID:17276777]

http://dx.doi.org/10.1016/S0140-6736(07)60193-7

10. Hijjawi JB, Kuiken TA, Lipschutz RD, Miller LA, Stubblefield KA, Dumanian GA. Improved myoelectric prosthesis control accomplished using multiple nerve transfers. Plast Reconstr Surg. 2006;118(7):1573-78. [PMID:17102730] http://dx.doi.org/10.1097/01.prs.0000242487.62487.fb

11. Miller LA, Stubblefield KA, Lipschutz RD, Lock BA, Kuiken TA. Improved myoelectric prosthesis control using targeted reinnervation surgery: a case series. IEEE Trans Neural Syst Rehabil Eng. 2008;16(1):46-50. [PMID:18303805] http://dx.doi.org/10.1109/TNSRE.2007.911817

12. Bouwsema H, van der Sluis CK, Bongers RM. Movement characteristics of upper extremity prostheses during basic goal-directed tasks. Clin Biomech (Bristol, Avon). 2010; 25(6):523-29. [PMID:20362374] http://dx.doi.org/10.1016/j.clinbiomech.2010.02.011

13. Doeringer JA, Hogan N. Performance of above elbow bodypowered prostheses in visually guided unconstrained motion tasks. IEEE Trans Biomed Eng. 1995;42(6):621-31.

[PMID:7790019]

http://dx.doi.org/10.1109/10.387202

14. Miller LA, Lipschutz RD, Stubblefield KA, Lock BA, Huang H, Williams TW 3rd, Weir RF, Kuiken TA. Control of a six degree of freedom prosthetic arm after targeted muscle reinnervation surgery. Arch Phys Med Rehabil. 2008; 89(11):2057-65. [PMID:18996233] http://dx.doi.org/10.1016/j.apmr.2008.05.016

15. Kuiken TA, Li G, Lock BA, Lipschutz RD, Miller LA, Stubblefield KA, Englehart KB. Targeted muscle reinnervation for real-time myoelectric control of multifunction artificial arms. JAMA. 2009;301(6):619-28. [PMID:19211469]

http://dx.doi.org/10.1001/jama.2009.116

16. Black N, Biden EN, Rickards J. Using potential energy to measure work related activities for persons wearing upper limb prostheses. Robotica. 2005;23(3):319-27. http://dx.doi.org/10.1017/S0263574704001341.

17. Murgia A, Kyberd PJ, Chappell PH, Light CM. Marker placement to describe the wrist movements during activities of daily living in cyclical tasks. Clin Biomech (Bristol, Avon). 2004;19(3):248-54. [PMID:15003339] http://dx.doi.org/10.1016/j.clinbiomech.2003.11.012

18. Williams S, Schmidt R, Disselhorst-Klug C, Rau G. An upper body model for the kinematical analysis of the joint chain of the human arm. J Biomech. 2006;39(13):2419-29. [PMID:16159659] http://dx.doi.org/10.1016/j.jbiomech.2005.07.023

19. van Andel CJ, Wolterbeek N, Doorenbosch CA, Veeger DH, Harlaar J. Complete 3D kinematics of upper extremity functional tasks. Gait Posture. 2008;27(1):120-27. [PMID:17459709] http://dx.doi.org/10.1016/j.gaitpost.2007.03.002

20. Wu G, van der Helm FC, Veeger HE, Makhsous M, Van Roy P, Anglin C, Nagels J, Karduna AR, McQuade K, Wang X, Werner FW, Buchholz B; International Society of Biomechanics. ISB recommendation on definitions of joint coordinate systems of various joints for the reporting of human joint motion-Part II: shoulder, elbow, wrist and hand. J Biomech. 2005;38(5):981-92. [PMID:15844264] http://dx.doi.org/10.1016/j.jbiomech.2004.05.042

21. Rau G, Disselhorst-Klug C, Schmidt R. Movement biomechanics goes upwards: from the leg to the arm. J Biomech. 2000;33(10):1207-16. [PMID:10899329] http://dx.doi.org/10.1016/S0021-9290(00)00062-2

22. Hermansson LM, Fisher AG, Bernspång B, Eliasson AC. Assessment of capacity for myoelectric control: a new Rasch-built measure of prosthetic hand control. J Rehabil Med. 2005;37(3):166-71. [PMID:16040474]

23. Carey SL, Jason Highsmith M, Maitland ME, Dubey RV. Compensatory movements of transradial prosthesis users during common tasks. Clin Biomech (Bristol, Avon). 2008; 23(9):1128-35. [PMID:18675497] http://dx.doi.org/10.1016/j.clinbiomech.2008.05.008

24. Carey SL, Dubey RV, Bauer GS, Highsmith MJ. Kinematic comparison of myoelectric and body powered prostheses while performing common activities. Prosthet Orthot Int. 2009;33(2):179-86. [PMID:19367522] http://dx.doi.org/10.1080/03093640802613229

25. Stein RB, Walley M. Functional comparison of upper extremity amputees using myoelectric and conventional prostheses. Arch Phys Med Rehabil. 1983;64(6):243-48. [PMID:6860093] 
JRRD, Volume 49, Number 8, 2012

Submitted for publication October 31, 2011. Accepted in revised form March 20, 2012.

This article and any supplementary material should be cited as follows:

Hebert JS, Lewicke J. Case report of modified Box and Blocks test with motion capture to measure prosthetic function. J Rehabil Res Dev. 2012;49(8):1163-74.

http://dx.doi.org/10.1682/JRRD.2011.10.0207

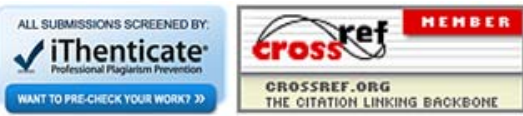

